\title{
Exciton localization mechanisms in wurtzite/zinc-blende GaAs nanowires
}

\author{
A. M. Graham, ${ }^{1}$ P. Corfdir,,${ }^{1,}$ M. Heiss, ${ }^{2}$ S. Conesa-Boj, ${ }^{2}$ E. Uccelli, ${ }^{2,3, \dagger}$ A. Fontcuberta i Morral, ${ }^{2}$ and R. T. Phillips ${ }^{1}$ \\ ${ }^{1}$ Cavendish Laboratory, University of Cambridge, J. J. Thomson Avenue, Cambridge CB3 OHE, United Kingdom \\ ${ }^{2}$ Laboratoire des Matériaux Semiconducteurs, Ecole Polytechnique Fédérale de Lausanne, 1015 Lausanne, Switzerland \\ ${ }^{3}$ Walter Schottky Institut and Physik Department, Technische Universität München, Garching, Germany
}

(Received 10 October 2012; published 8 March 2013)

\begin{abstract}
We investigate the emission properties of excitons in GaAs nanowires containing quantum disks formed by structural alternation between the zinc-blende and wurtzite phases, by means of temperature-dependent photoluminescence. At $10 \mathrm{~K}$ the emission from an ensemble of disks is distributed in a band of full width at half maximum $\sim 30 \mathrm{meV}$, whereas the emission linewidth for a single disk is $700 \mu \mathrm{eV}$. While the disk ensemble emission exhibits an S-shaped temperature dependence, the emission from single quantum disks follows the temperature dependence of the band gap over the whole temperature range. This indicates that intradisk exciton localization on impurities is negligible and that increasing the temperature induces a transfer of excitons from narrow to thick disks along the length of the wires. Our observations of the emission linewidth for single crystal-phase quantum disks show a scattering rate of excitons with acoustic phonons eight times larger than the values usually reported for $(\mathrm{Al}, \mathrm{Ga}) \mathrm{As} / \mathrm{GaAs}$ quantum wells. This large scattering rate demonstrates that the electron effective mass in wurtzite GaAs is much heavier than in zinc-blende GaAs and is evidence of coupling between the $\Gamma_{7}$ and $\Gamma_{8}$ conduction bands of wurtzite GaAs.
\end{abstract}

DOI: 10.1103/PhysRevB.87.125304

PACS number(s): 78.67.Uh, 78.55.Cr, 78.67.De

While most bulk non-nitride III-V semiconductors crystallize in the zinc-blende $(\mathrm{ZB})$ phase, nanowires can crystallize as wurtzite (WZ), ZB, or polytypic combinations of these. ${ }^{1-5}$ Polytypism is usually considered detrimental in bulk semiconductors, ${ }^{6,7}$ but recent reports have revealed the possibility of tuning the crystal phase along the length of III-V nanowires. ${ }^{1,2,8-10}$ A new method of confining charge carriers can then be achieved by the alternation of ZB and WZ segments along the length of the wires. ${ }^{5}$ In these so-called crystal-phase quantum structures, ${ }^{5}$ electrons are confined to the $\mathrm{ZB}$ and holes to the $\mathrm{WZ}$ segments of the wires, forming type-II heterostructures. ${ }^{11}$ Single-photon emission at $4 \mathrm{~K}$ from InP individual crystal-phase quantum disks has recently been reported ${ }^{5}$ and polarization-resolved photoluminescence (PL) experiments performed on GaAs nanowires in the 4-20 K temperature $(T)$ range have revealed that the ground state of the excitons bound to crystal-phase quantum disks is formed by heavy holes. ${ }^{12}$ Numerous features of these crystalphase quantum disks have still to be fully understood, and in particular, the mechanisms of exciton localization and delocalization occurring in polytypic wires at intermediate $T$ remain unclear.

In this study, we investigate the localization mechanisms of excitons in polytypic GaAs wires grown by molecular beam epitaxy through $T$-dependent photoluminescence between 4 and $170 \mathrm{~K}$. At low $T$, excitons are bound to crystal-phase quantum disks. Comparing the $T$ dependence of the PL from ensemble and single nanowires, we show that intradisk localization phenomena are negligible and that exciton hopping from narrow to thicker disks is activated for $T$ typically above $20 \mathrm{~K}$. From the $T$ dependence of the exciton linewidth in single quantum disks, we finally demonstrate that the scattering of excitons with acoustic phonons is much more efficient in crystal-phase quantum disks than in ( $\mathrm{Al}, \mathrm{Ga}) \mathrm{As} / \mathrm{GaAs}$ quantum wells.

GaAs nanowires have been grown by molecular beam epitaxy (MBE) on a $\mathrm{SiO}_{2}$-coated (111)B GaAs substrate. As reported previously in Ref. 13, the growth temperature was set to $630{ }^{\circ} \mathrm{C}$, and the As partial pressure was continuously varied from $8.8 \times 10^{-7}$ to $3.0 \times 10^{-6}$ mbar. The resulting wires are typically a few micrometers long with a diameter of 100-200 nm. Since dangling bonds in GaAs induce states close to the middle of the gap ${ }^{14}$ the wires were capped with an $(\mathrm{Al}, \mathrm{Ga}) \mathrm{As}$ shell to reduce the efficiency of nonradiative recombination via surface states. ${ }^{15,16}$ High-resolution transmission electron microscopy (HRTEM) has been performed using a Schottky field emission gun working at $300 \mathrm{kV}$ with a point-to-point resolution of $0.17 \mathrm{~nm}$, and scanning electron microscopy has been carried out with a microscope operating at $7 \mathrm{kV}$. Figures 1(a) and 1(b) show typical HRTEM micrographs of sections of the nanowire presenting a mixture of ZB and WZ. The thickness of the sections (disks) typically varies between 1 and $10 \mathrm{~nm}$. The wires studied are polytypic, with an equivalent average volume fraction of the ZB structure of at least $80 \%$. In this sample, the density of twins varies between 1 and $500 \mu \mathrm{m}^{-1}$ along the nanowire axis. The HRTEM micrographs of Figs. 1(a) and 1(b) correspond to a region with a higher density of polytypism. The PL of the GaAs wires was excited with a He:Ne laser (excitation wavelength $\lambda=632.8 \mathrm{~nm}$ ). PL experiments on ensembles of as-grown wires were performed in an exchange gas cryostat. We estimate the diameter of the excitation spot to be $80 \mu \mathrm{m}$ and the excitation power density was kept between 0.01 and $5 \mathrm{~W} / \mathrm{cm}^{2}$. We rule out that the detected PL could originate from the (111)B GaAs substrate, as (i) the wire distribution in the as-grown sample is dense [Fig. 1(c)] and (ii) similar results were obtained on ensembles of wires dispersed on a Si substrate. In order to perform PL measurements on single wires, we mechanically dispersed the wires onto an Au grid fixed to a $p$-doped Si substrate [Fig. 1(d)]. The sample was placed in a continuous flow coldfinger cryostat mounted on a nanopositioning stage with minimum motor step of $100 \mathrm{~nm}$. Micro-PL experiments were performed in a confocal system using a high-numerical-aperture objective ( $0.5 \mathrm{NA})$, allowing 

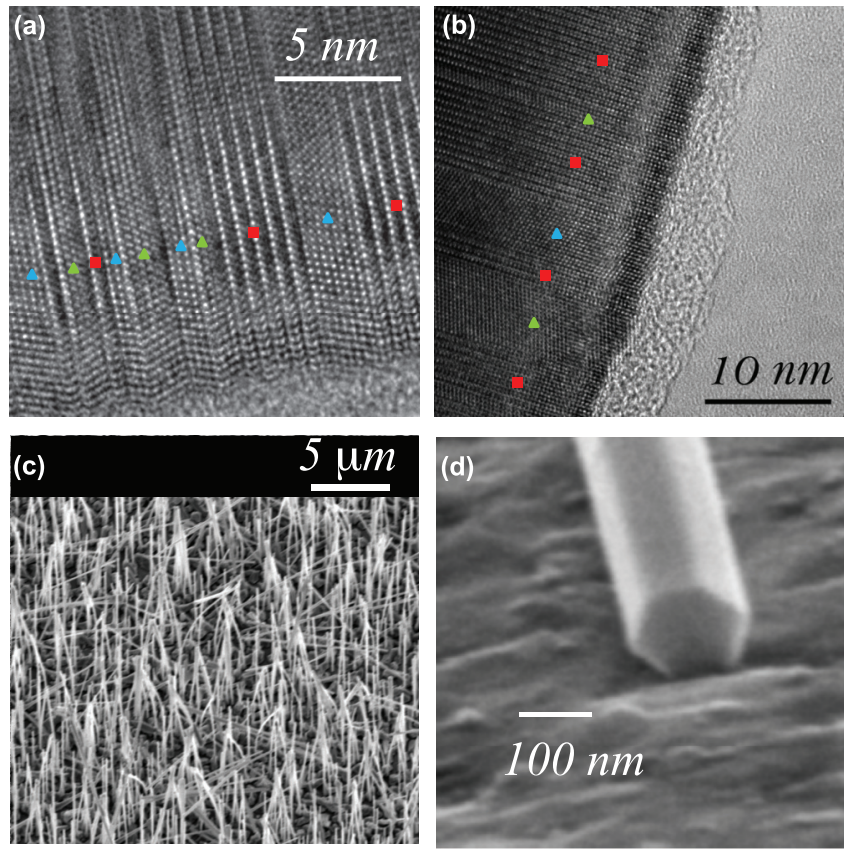

FIG. 1. (Color online) (a) and (b) High-resolution TEM micrographs viewed along the [110] zone axis for two different regions of a nanowire. (a) and (b) show a mixture of zinc-blende (blue and green triangles correspond to zinc-blende phase domains with different orientation) and wurtzite phase (red squares). (c) Scanning electron micrograph of a GaAs nanowires ensemble. (d) Scanning electron micrograph of a GaAs nanowire dispersed on a Si substrate.

for focusing of the laser beam down to a $1.5-\mu \mathrm{m}$-diameter spot. The wires were excited with optical power of $4 \mathrm{nW}$. The wire emission was analyzed with a spectrograph of focal length $640 \mathrm{~mm}$, using either a 600 or 1200 grooves per mm grating, followed by detection on a Peltier-cooled CCD.

Figure 2(a) shows PL spectra taken between 10 and $170 \mathrm{~K}$ with an excitation density of $5 \mathrm{~W} / \mathrm{cm}^{2}$ for an ensemble of as-grown GaAs wires. The PL at $10 \mathrm{~K}$ is dominated by a broad emission centered at $1.483 \mathrm{eV}$ with full width at half maximum
(FWHM) of $30 \mathrm{meV}$. On the high-energy side of this band, we observe additional shoulders at 1.496 and $1.510 \mathrm{eV}$ as well as a narrow emission line $(\mathrm{FWHM}=2 \mathrm{meV})$ centered at $1.516 \mathrm{eV}$. Although the precise value of the band gap of WZ GaAs is still debated, ${ }^{8,13,17-23}$ recent resonant Raman scattering experiments performed on $\mathrm{WZ}$ GaAs nanowires have indicated that the $\mathrm{WZ}$ and the $\mathrm{ZB}$ phases of GaAs have a similar band gap. ${ }^{8}$ As a consequence, we attribute the emission at $1.516 \mathrm{eV}$ to the recombination of excitons in purely $\mathrm{ZB}$ or WZ segments. We also ascribe the $2 \mathrm{meV}$ inhomogeneous broadening of the $1.516 \mathrm{eV}$ line to a possible slight difference between the band gaps of $\mathrm{WZ}$ and $\mathrm{ZB} \mathrm{GaAs,}$ as well as to the variation of strain state along the wires, as revealed by micro-Raman experiments on GaAs nanowires. ${ }^{24}$ Regarding the broad PL at $1.483 \mathrm{eV}$ and its higher-energy shoulders, their origin can be twofold. These lines correspond in energy to the emission from the polytypic regions of the wires, i.e., to the emission from the ensemble of crystal-phase quantum disks, and also to the energy of transitions involving carbon impurities. ${ }^{20}$ When $T$ is increased, one can note from Fig. 3 that the emission line at $1.516 \mathrm{eV}$ continuously redshifts, following the empirical Varshni relation $E(T)=E(0)-\alpha T^{2} /(T+\beta){ }^{25}$ The $T$ dependence of the emission energy of this line can be satisfactorily fitted using $\alpha=0.85 \mathrm{meV} / \mathrm{K}$ and $\beta=650 \mathrm{~K}$, which are in the range of the values reported for bulk $\mathrm{ZB}$ GaAs. $^{26}$ Concerning the broad emission at $1.483 \mathrm{eV}$ at $4 \mathrm{~K}$, the $T$ dependence of its peak energy does not follow the Varshni formula (Fig. 2). This is in contradiction to what has been reported for conduction-band-acceptor transitions related to carbon, ${ }^{27}$ and we therefore ascribe the band at $1.483 \mathrm{eV}$ to the emission from the polytypic regions of the wires. We attribute the $30 \mathrm{meV}$ inhomogeneous broadening of the $1.483 \mathrm{eV}$ emission to the distribution of quantum disk thicknesses and to the variation of disk-disk coupling schemes in the wire ensemble. ${ }^{13,28}$

In Fig. 3 we present the $T$ dependence of the emission energy from the disk ensemble and from the purely $\mathrm{ZB}$ and WZ segments for various excitation densities. For an excitation density of $5 \mathrm{~W} / \mathrm{cm}^{2}$, the emission from the disk ensemble first redshifts by $10 \mathrm{meV}$ between 10 and $120 \mathrm{~K}$,
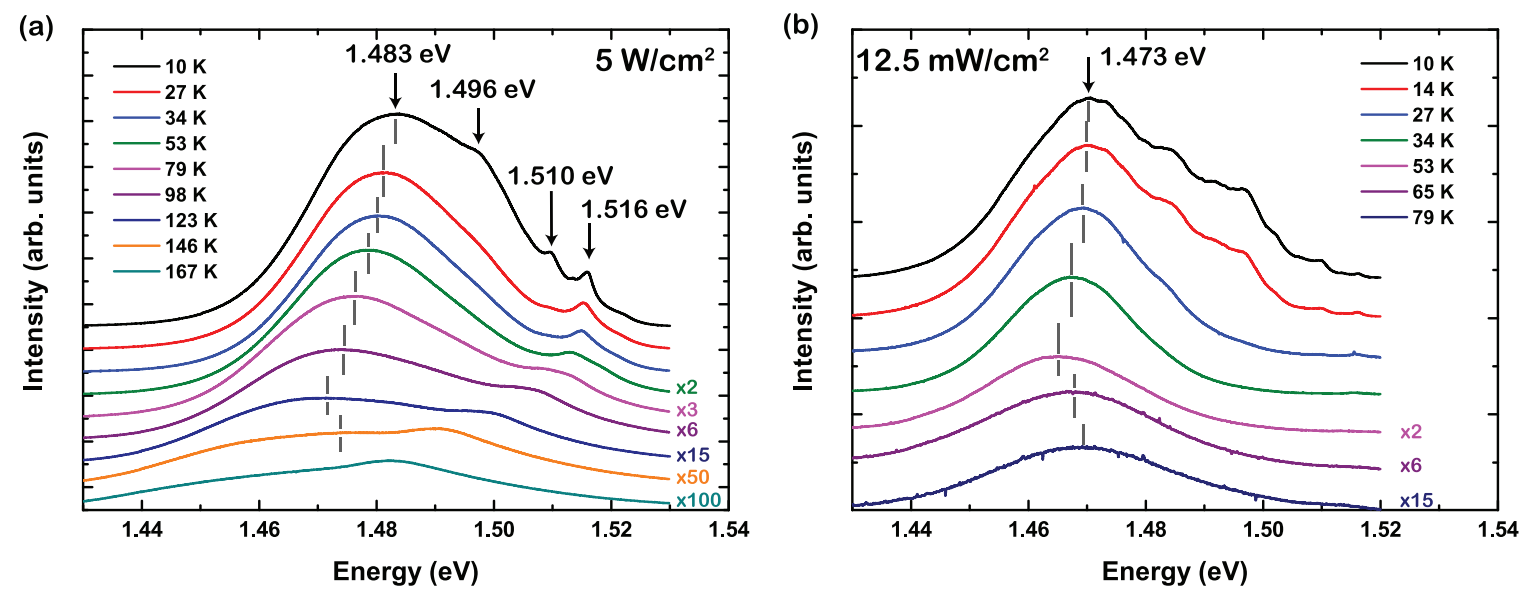

FIG. 2. (Color online) (a) Photoluminescence from an ensemble of polytypic GaAs nanowires as a function of temperature. The power excitation density is set to $5 \mathrm{~W} / \mathrm{cm}^{2}$ (a) and $12.5 \mathrm{~mW} / \mathrm{cm}^{2}$ (b). Spectra have been shifted vertically for clarity. The gray dashed lines are guides to the eye showing the S-shaped temperature dependence of the emission from the ensemble of disks. 


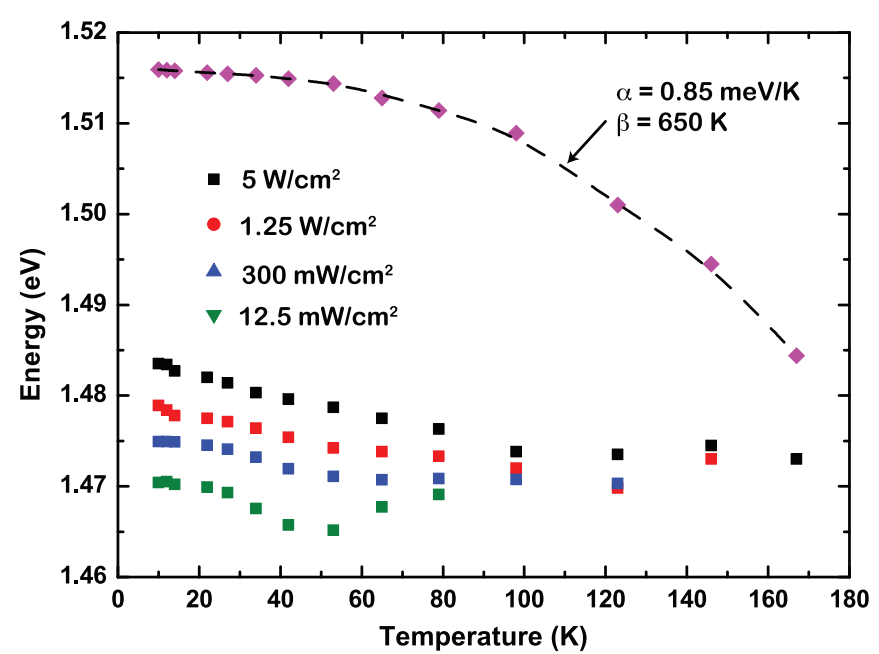

FIG. 3. (Color online) Temperature dependence of the quantum disks ensemble peak emission energy (squares) for an excitation power density between 0.0125 and $5 \mathrm{~W} / \mathrm{cm}^{2}$. In this range of excitation power density, the emission energy from the purely zinc-blende and wurtzite segments (diamonds) does not depend on the excitation. The dashed line shows the result of Varshni's empirical model fit, using $\alpha=0.85 \mathrm{meV} / \mathrm{K}$ and $\beta=650 \mathrm{~K}$.

then blueshifts by $\sim 1 \mathrm{meV}$ from 120 to $140 \mathrm{~K}$ before finally redshifting again. This so-called "S-shaped temperature dependence" is typical of disordered systems and has, for instance, already been reported for (In,Ga)N/GaN quantum wells, ${ }^{29}$ (Ga,In,N,As)/(Ga,As,N) quantum wells, ${ }^{30}$ or for basal stacking faults in wide band-gap semiconductors. ${ }^{31-33} \mathrm{We}$ attribute the initial redshift observed here to the detrapping of excitons from the shallowest traps in favor of the deepest ones. This is confirmed by the vanishing of the high-energy shoulders of the quantum disk emission band when $T$ is increased from 10 to typically $35 \mathrm{~K}$ (Fig. 2). For higher $T$, excitons have enough thermal energy to fill higher-energy localized states, resulting in a blueshift of the emission together with an increase in emission intensity from the high-energy tail of the emission band (Fig. 2).

Temperature-dependent PL experiments taken with lower excitation densities fully corroborate our picture [Figs. 2(b) and 3]. While the emission energy from the purely $\mathrm{WZ}$ and $\mathrm{ZB}$ regions of the wires remains constant when the excitation power is decreased from 5 to $0.01 \mathrm{~W} / \mathrm{cm}^{2}$, we observe that (i) the peak emission energy of the crystal-phase quantum disk ensemble redshifts by $10 \mathrm{meV}$ and (ii) the high-energy tail of the disk ensemble PL collapses, which we attribute to reduced localized state filling. ${ }^{30}$ As shown in Fig. 3, we also observe that the higher the excitation density, the less pronounced the $S$ shaped $T$ dependence of the quantum disks' emission energy. A similar "S-shape bleaching" has recently been reported by Hammersley et al. for (In,Ga)N/GaN quantum wells and has been ascribed to the saturation of available localized states at high excitation densities. ${ }^{34}$ We note, finally, that for excitation densities higher than $10 \mathrm{~W} / \mathrm{cm}^{2}$, the PL from the $\mathrm{WZ}$ and $\mathrm{ZB}$ segments redshifts and broadens due to local laser-induced heating of the sample. ${ }^{13}$

Although the $T$ - and excitation-dependent experiments discussed above provide evidence of exciton localization along the wire length, the origin of the $\mathrm{S}$-shaped $T$ dependence observed in Fig. 3 remains to be fully clarified. In usual III-V semiconductor quantum wells, exciton localization occurs as a result of well-width fluctuation ${ }^{35}$ or on potential fluctuations induced by alloy disorder in the well or in the barriers. ${ }^{29,36}$ The present $\mathrm{GaAs} / \mathrm{GaAs}$ homostructures are of interest because any potential fluctuations from alloy disorder are excluded. Furthermore, we can eliminate thickness fluctuations of the quantum disks, as these have been shown to have interfaces flat to within a monolayer. ${ }^{5}$ The S-shaped $T$ dependence of the emission from the disk ensemble may originate from two effects. First, although crystal-phase quantum disks are clean structures, in the sense that they are free of well-width fluctuation and alloy disorder, there are still some impurities distributed in or away from the disk that can trap the excitons. These impurities not only contribute to the QW emission linewidth ${ }^{37}$ but also can give rise, when the interface roughness is negligible, to additional emission lines at low $T .^{38,39}$ Due to the type-II band alignment between $\mathrm{WZ}$ and $\mathrm{ZB}$ GaAs, ${ }^{11}$ the spatial extent of the exciton wave function is large, making it sensitive to point defects distributed in the vicinity of the disks. ${ }^{28}$ Such a localization process has, for instance, been observed for basal stacking faults in $\mathrm{GaN}$ or $\mathrm{ZnO}$, as discussed in Refs. 33 and 40. The second possible origin for the $S$-shaped $T$ dependence of the quantum disk emission is the thermal activation of a disk-to-disk hopping mechanism for excitons. The conduction and valence-band offsets between cubic and hexagonal GaAs are small enough ${ }^{11,13}$ for the thermal escape of excitons to be activated at intermediate $T$. The initial redshift observed in Fig. 3 would then simply result from the escape of carriers from the narrowest disks and their subsequent trapping by thicker ones.

In order to elucidate which of the mechanisms described above (intra quantum disk localization or exciton hopping along the wire length) is the dominant one, we have performed $T$-dependent micro-PL experiments on single polytypic GaAs nanowires. In Fig. 4(a) we display the PL spectra at $4 \mathrm{~K}$ for three different wires. The emission spectra from single nanowires consist of narrow emission lines centered between 1.460 and $1.510 \mathrm{eV}$. The narrowest lines observed can be fitted with Lorentzian curves with FWHM of $\sim 700 \mu \mathrm{eV}$ [Fig. 4(a)] and we ascribe them to the recombination of excitons confined to quantum disks located in wire segments with a low twin density. We emphasize that the emission linewidths reported here for single GaAs crystal-phase quantum disks are similar to those reported for thick MBE-grown (Al,Ga)As/GaAs quantum wells (see, for instance, Ref. 41, where a linewidth of $500 \mu \mathrm{eV}$ is reported for a $20 \mathrm{~nm}$-thick $\mathrm{Al}_{0.3} \mathrm{Ga}_{0.7} \mathrm{As} / \mathrm{GaAs}$ single quantum well). When $T$ is increased from 4 to $50 \mathrm{~K}$, the emission from single crystal-phase quantum disks continuously redshifts, following the band-gap $T$ dependence [Fig. 4(b)]. Note that for each temperature step, we had to compensate for the thermal drift of the sample holder. This was achieved by reoptimizing the position of the sample in order to maximize the PL signal from the disk of interest, a procedure that prevents us from making a quantitative analysis of the $T$ dependence of the single disk emission intensity. In addition, despite this realignment procedure, the disk emission intensity decreases drastically, which prevents tracking of the emission line of single disks for $T$ higher than $\sim 50 \mathrm{~K}$. The 
(a)

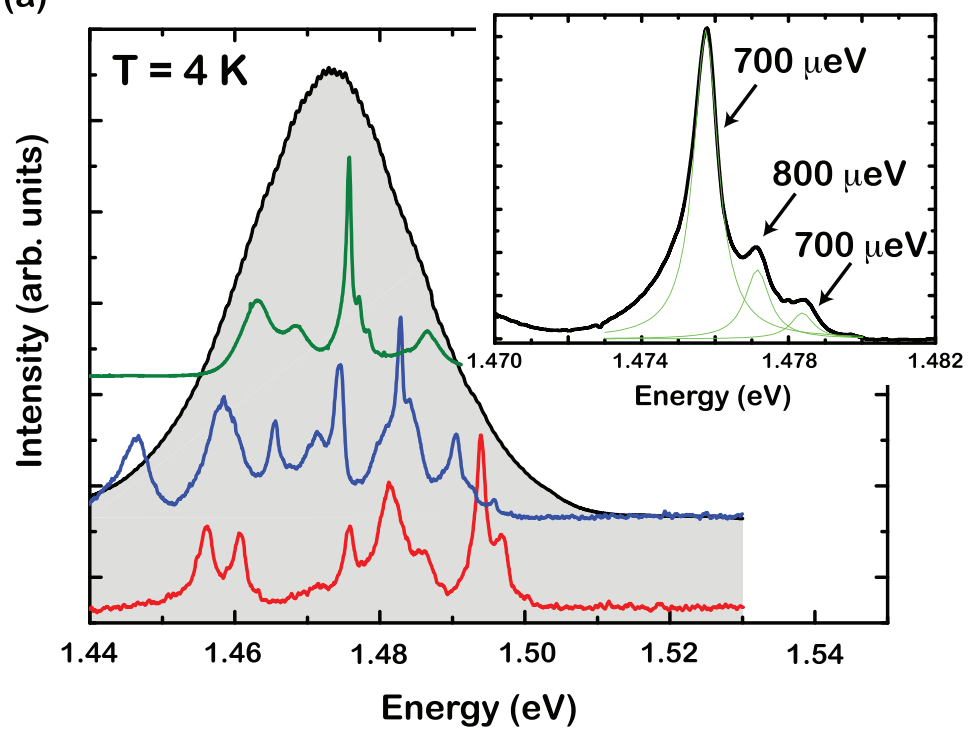

(b)

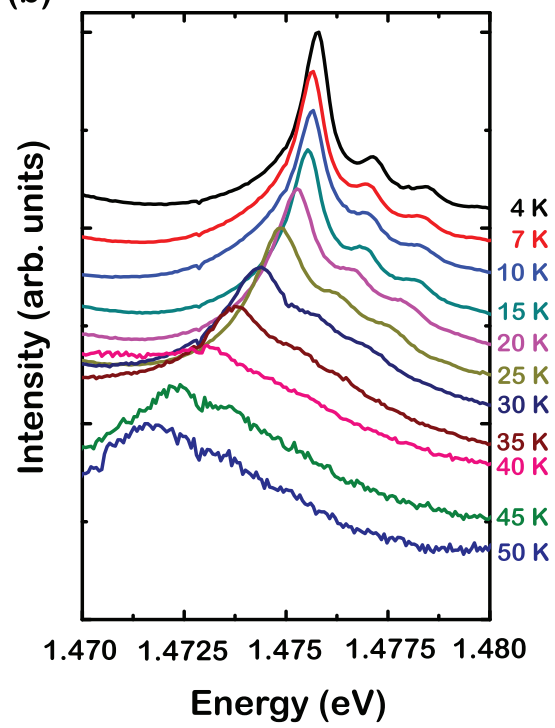

FIG. 4. (Color online) (a) Microphotoluminescence spectra taken at $4 \mathrm{~K}$ on single polytypic GaAs nanowires with an excitation power of $4 \mathrm{nW}$. Spectra have been shifted vertically for clarity. The gray shaded area shows the emission from an ensemble of wires. Inset: high-resolution spectrum showing the emission from single disks, together with the result of a fitting with a Lorentzian line shape. The emission from single disks exhibits a full width at half maximum of $700 \mu \mathrm{eV}$. (b) Temperature-dependent PL on single quantum disks. In contrast with the behavior measured for the disk ensemble, the emission energy for a single disk follows the temperature dependence of the band gap.

purely monotonic $T$ dependence for the emission energy of single quantum disks demonstrates that in contrast to what was observed in WZ/ZB homostructures for wide-band-gap semiconductors, intradisk localization effects are negligible in GaAs. This difference probably results from the small effective masses and the large dielectric constant of GaAs, compared to $\mathrm{ZnO}$ and $\mathrm{GaN}$, both of which diminish the importance of excitonic effects. These observations strongly suggest that we may attribute the S-shaped $T$ dependence displayed in Fig. 3 to the transfer of excitons from narrow to thicker disks distributed along the length of the wires. As further support for this, we note the increase of the intensity ratio between the emission from the purely ZB (or WZ) segments and that from the disk ensemble [Fig. 2(a)] for $T$ above $20 \mathrm{~K}$. This demonstrates that at $20 \mathrm{~K}$, the escape of excitons from the quantum disks to the barriers is activated, enabling the transfer of excitons from narrow to thicker disks, i.e., from high- to low-energy emitting disks.

The evolution with $T$ of the emission linewidth $\hbar \Gamma$ brings additional information about the scattering of excitons with phonons (Fig. 5). For $T$ below $40 \mathrm{~K}, \hbar \Gamma$ increases monotonically, with a very nearly linear dependence on $T$. The exciton linewidth follows $\hbar \Gamma(T)=\hbar \Gamma_{0}+\hbar \Gamma_{a c} T+\hbar \Gamma_{\mathrm{LO}} N_{\mathrm{LO}}(T)$, where $\hbar \Gamma_{0}$ is the exciton broadening at $0 \mathrm{~K}, \Gamma_{a c}$ is the scattering rate of excitons with acoustic phonons, $\Gamma_{L O}$ characterizes the coupling strength of excitons with longitudinal optical (LO) phonons, and $N_{L O}$ is the LO-phonon occupancy. ${ }^{42}$ Despite the fact that the Fröhlich interaction is stronger in GaAs nanowires than in bulk material, ${ }^{43}$ the LO-phonon energy is $36 \mathrm{meV}$ (Ref. 44) and we can therefore safely neglect the influence of LO phonons on the emission broadening for $T<50 \mathrm{~K} .{ }^{41}$ From linear fits to the $T$ dependence of the emission linewidth of several quantum disks, we find that $\hbar \Gamma_{a c}$ ranges between 9 and
$41 \mu \mathrm{eV} / \mathrm{K}$ (Fig. 5). In (Al,Ga)As/GaAs quantum wells grown along [001], $\hbar \Gamma_{a c}$ usually falls between 1 and $10 \mu \mathrm{eV} / \mathrm{K}^{41,45,46}$ Slightly enhanced exciton-acoustic phonon scattering rates (up to $12 \mu \mathrm{eV} / \mathrm{K}$ ) have been observed in (In,Ga)As/GaAs quantum wires and have been ascribed to the effect of the lateral confinement. ${ }^{47}$ This nevertheless does not apply to the GaAs quantum disks studied here, since the diameter of the wires is typically 100-200 nm [Fig. 1(c)] and the effect of

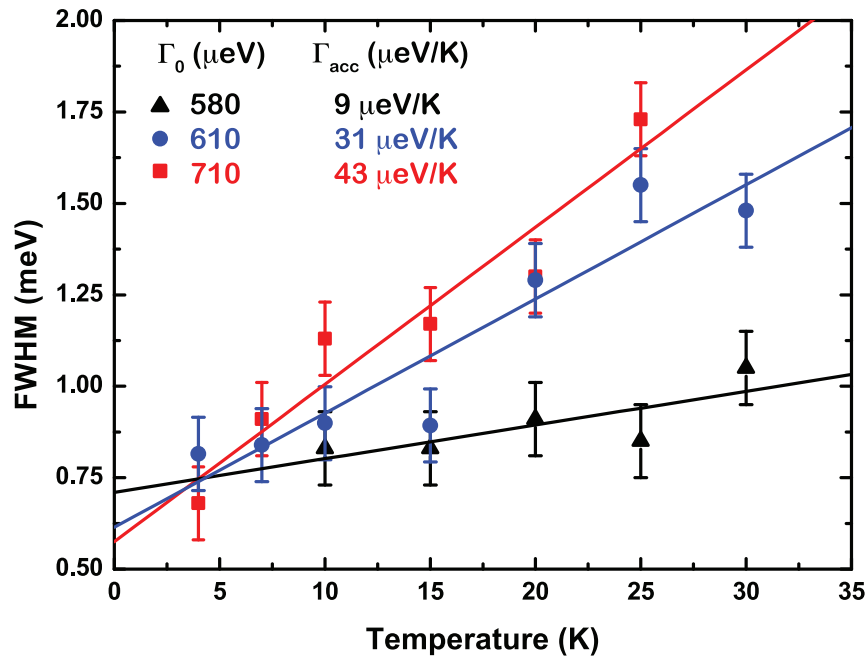

FIG. 5. (Color online) Temperature dependence of the FWHM of photoluminscence emission from three single WZ/ZB quantum disks embedded in three different wires. Solid lines show linear fits that allow extraction of both the disk emission linewidth at $0 \mathrm{~K}$ and the scattering rate of excitons with acoustic phonons $\left(\Gamma_{0}\right.$ and $\Gamma_{a c}$, respectively). 


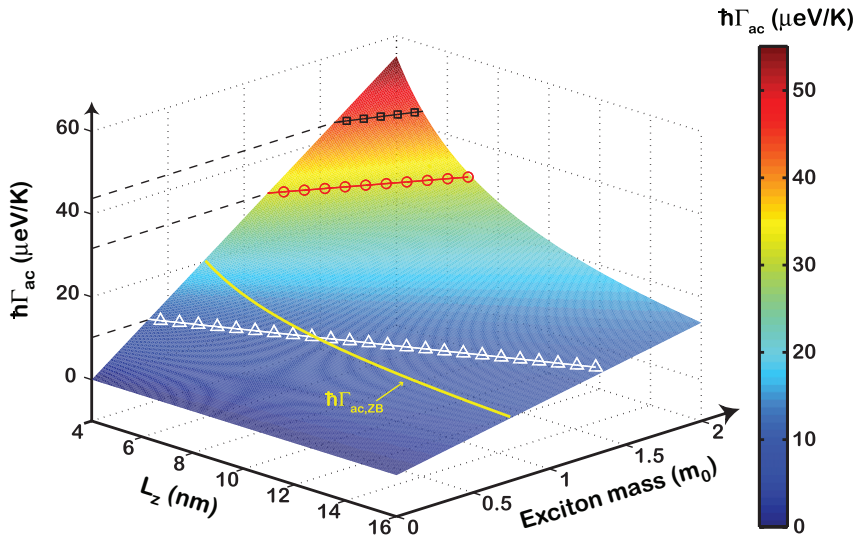

FIG. 6. (Color online) Variation of the exciton-acoustic phonon scattering rate $\Gamma_{a c}$ with respect to the in-plane exciton translational mass $(M)$ and the extent of the exciton wave function along the confinement axis $\left(L_{z}\right) . \Gamma_{a c, Z B}$ (yellow line) is obtained using $M=0.71 m_{0}$ and is the lower limit for $\hbar \Gamma_{a c}$ in GaAs crystal-phase quantum disks. Squares, circles, and triangles show the scattering rates obtained from Fig. 5 and correspond to $\hbar \Gamma_{a c}=43,31$, and $9 \mu \mathrm{eV} / \mathrm{K}$, respectively.

lateral confinement can therefore be neglected. We emphasize that we did not notice any correlation of $\hbar \Gamma_{a c}$ with the emission energy of the quantum disk.

In order to understand the physical origin of the large values of $\hbar \Gamma_{a c}$ measured in GaAs crystal-phase quantum disks, we compute in the following the exciton-acoustic phonon scattering rate. The large uncertainties on the conduction- and valence-band offsets between WZ and ZB GaAs (Refs. 11, 13,21, and 48) hinder the exact calculation of $\hbar \Gamma_{a c}$ using the model of Ref. 49. An alternative approach has been proposed in Refs. 50 and 51, which gives for the exciton-acoustic phonon scattering rate in a $\mathrm{QW}$,

$$
\hbar \Gamma_{a c}=\frac{k_{B} D^{2} M}{L_{z} \hbar^{2} u^{2} \rho} m
$$

where $D, M, u$, and $\rho$ are the interband hydrostatic deformation potential, the in-plane exciton mass, the longitudinal sound velocity, and the density, respectively. For quantum wells with infinite barriers, $L_{z}$ is the quantum well thickness. ${ }^{50}$ In the case of crystal-phase quantum disks, however, the exciton wave function spreads significantly into the barriers ${ }^{28}$ and $L_{z}$ characterizes the spatial extension of the exciton wave function along the confinement axis. ${ }^{51}$ In Fig. 6 we plot the evolution of $\hbar \Gamma_{a c}$ with respect to $M$ and $L_{z}$, taking $\rho=$ $5316.5 \mathrm{~g} / \mathrm{cm}^{3}$ (Ref. 52), $u=4726 \mathrm{~m} / \mathrm{s}$ (Ref. 53), and $D=$ $8.5 \mathrm{eV}^{26,54}$ In agreement with recent $a b$ initio calculations, we assume $D$ to be the same for both the WZ and the $\mathrm{ZB}$ phases of GaAs. ${ }^{55}$ In the $\mathrm{ZB}$ domains, the in-plane exciton mass is $M_{\mathrm{ZB}}=m_{e}+m_{h,[1 \overline{1} 0]}=0.71 m_{0}$, where $m_{h,[1 \overline{1} 0]}$ is the hole effective mass along the [110] direction (we use the Luttinger parameters given in Ref. 26). Concerning the exciton translational mass in the WZ segments $\left(M_{\mathrm{WZ}}\right)$, its value remains uncertain due to the lack of report for the effective hole mass along the nonpolar directions. However, we can safely assume that $M_{\mathrm{WZ}}$ is equal to or larger than
$M_{\mathrm{ZB}}$, the possible coupling of the $\Gamma_{7}$ conduction band with the (heavier) $\Gamma_{8}$ band leading to an increase in electron effective mass. ${ }^{13,56,57}$ We mention, for instance, that an electron effective mass of $1.092 m_{0}$ has been reported in Ref. 13 for WZ GaAs. Now, due to the type-II band alignment between $\mathrm{WZ}$ and $\mathrm{ZB}$ GaAs, the wave function of an exciton bound to a $\mathrm{ZB}$ (WZ) disk spreads significantly inside the WZ (ZB) barriers: ${ }^{28}$ The exciton-phonon scattering $\hbar \Gamma_{a c, Z B}$ computed using $M=M_{\mathrm{ZB}}$ provides, therefore, a lower limit for $\hbar \Gamma_{a c}$ in GaAs crystal-phase quantum disks.

In Fig. 6 we display $\hbar \Gamma_{a c, \mathrm{ZB}}$ together with the experimental $\hbar \Gamma_{a c}$ deduced from Fig. 5 for three distinct quantum disks. On the one hand, disks exhibiting $\hbar \Gamma_{a c}$ of the order of $10 \mu \mathrm{eV} / \mathrm{K}$ correspond to excitons with $M$ close to $M_{\mathrm{ZB}}$, which leads to the conclusion that the electron wave function of these excitons spreads mainly in segments with $\mathrm{ZB}$ phase. This is, e.g., the case for excitons localized on thick $\mathrm{ZB}$ inclusions. On the other hand, we attribute $\hbar \Gamma_{a c}$ as large as $40 \mu \mathrm{eV} / \mathrm{K}$ to quantum disk excitons with large probability of the electron being found in the WZ phase. This occurs, for instance, when an exciton is bound to a 3-monolayer-thick ZB segment. ${ }^{28}$ This implies that the exciton translational mass in $\mathrm{WZ}$ GaAs is much heavier than $M_{\mathrm{ZB}}$, supporting qualitatively the assumption of coupling between the $\Gamma_{7}$ and $\Gamma_{8}$ conduction bands in WZ GaAs. Finally, we note that a heavier electron effective mass in the GaAs WZ phase also results in a smaller exciton Bohr radius, which is in qualitative agreement with the trend observed for $L_{z}$ in Fig. 6.

In conclusion, we have measured the temperature dependence of the photoluminescence from GaAs crystal-phase quantum disks embedded in GaAs nanowires. The emission at $10 \mathrm{~K}$ from crystal-phase quantum disks in an ensemble of wires exhibits inhomogeneous broadening of $30 \mathrm{meV}$, and the emission linewidth from single quantum disks is typically $700 \mu \mathrm{eV}$. When temperature is increased, the energy of emission from an ensemble follows an S-shaped temperature dependence, which we interpret as evidence of exciton redistribution among states within the polytypic wires. In contrast, the emission lines from single quantum disks redshift monotonically, following the temperature dependence of the band gap. This not only indicates that intradisk exciton localization on impurities can be neglected, but also allows us to ascribe the S-shaped temperature dependence observed for the emission from the ensemble of disks to the thermal activation of disk-to-disk exciton hopping mechanisms along the length of the wires. We observe in single crystal-phase quantum disks a coefficient for the temperature linear term in the exciton linewidth up to $41 \mu \mathrm{eV} / \mathrm{K}$, corresponding to an acoustic phonon scattering rate eight times larger than the values typically reported for (Al,Ga)As/GaAs quantum wells. We attribute this efficient scattering to the large electron effective mass in wurtzite GaAs, as a result of the coupling between the $\Gamma_{7}$ and $\Gamma_{8}$ conduction bands of wurtzite GaAs.

We acknowledge financial support from the Poynton Cambridge Australia Scholarship and from the European Union Seventh Framework Program under grant agreement No. 265073. A.F.iM. and E.U. acknowledge funding through the Marie Curie Excellence grant SENFED. S.C.B. thanks S.N.F. for funding through the Marie-Heim Vögtlin scheme. 
*Corresponding author: pmc53@cam.ac.uk

${ }^{\dagger}$ Present address: IBM Zurich Research Laboratory, CH-8803 Rüschlikon, Switzerland.

${ }^{1}$ P. Caroff, K. A. Dick, J. Johansson, M. E. Messing, K. Deppert, and L. Samuelson, Nat. Nanotechnol. 4, 50 (2009).

${ }^{2}$ R. E. Algra, M. A. Verheijen, M. T. Borgström, L.-F. Feiner, G. Immink, W. J. P. van Enckevort, E. Vlieg, and E. P. A. M. Bakkers, Nature (London) 456, 369 (2008).

${ }^{3}$ J. Bao, D. C. Bell, F. Capasso, J. B. Wagner, T. Mårtensson, J. Trägårdh, and L. Samuelson, Nano Lett. 8, 836 (2008).

${ }^{4}$ D. Spirkoska, J. Arbiol, A. Gustafsson, S. Conesa-Boj, F. Glas, I. Zardo, M. Heigoldt, M. H. Gass, A. L. Bleloch, S. Estrade, M. Kaniber, J. Rossler, F. Peiro, J. R. Morante, G. Abstreiter, L. Samuelson, and A. Fontcuberta i Morral, Phys. Rev. B 80, 245325 (2009).

${ }^{5}$ N. Akopian, G. Patriarche, L. Liu, J.-C. Harmand, and V. Zwiller, Nano Lett. 10, 1198 (2010).

${ }^{6}$ K. H. Baik, Y. G. Seo, S. K. Hong, S. Lee, J. Kim, J. S. Son, and S. M. Hwang, IEEE Photonics Technol. Lett. 22, 595 (2010).

${ }^{7}$ C. Thelander, P. Caroff, S. Plissard, A. W. Dey, and K. A. Dick, Nano Lett. 11, 2424 (2011).

${ }^{8}$ B. Ketterer, M. Heiss, E. Uccelli, J. Arbiol, and A. Fontcuberta i Morral, ACS Nano 5, 7585 (2011).

${ }^{9}$ S. Plissard, K. A. Dick, G. Larrieu, S. Godey, A. Addad, X. Wallart, and P. Caroff, Nanotechnology 21, 385602 (2010).

${ }^{10}$ K. A. Dick, C. Thelander, L. Samuelson, and P. Caroff, Nano Lett. 10, 3494 (2010).

${ }^{11}$ M. Murayama and T. Nakayama, Phys. Rev. B 49, 4710 (1994).

${ }^{12}$ D. Spirkoska, A. L. Efros, W. R. L. Lambrecht, T. Cheiwchanchamnangij, A. Fontcuberta i Morral, and G. Abstreiter, Phys. Rev. B 85, 045309 (2012).

${ }^{13}$ J. M. Ménard, A. E. Mattacchione, H. M. van Driel, C. Hautmann, and M. Betz, Phys. Rev. B 82, 045303 (2010).

${ }^{14}$ D. D. Nolte, Solid State Electron. 33, 295 (1990).

${ }^{15}$ O. Demichel, M. Heiss, J. Bleuse, H. Mariette, and A. Fontcuberta i Morral, Appl. Phys. Lett. 97, 201907 (2010)

${ }^{16}$ C.-C. Chang, C.-Y. Chi, M. Yao, N. Huang, C.-C. Chen, J. Theiss, A. W. Bushmaker, S. LaLumondiere, T.-W. Yeh, M. L. Povinelli, C. Zhou, P. D. Dapkus, and S. B. Cronin, Nano Lett. 12, 4484 (2012).

${ }^{17}$ B. V. Novikov, S. Yu. Serov, N. G. Filosofov, I. V. Shtrom, V. G. Talalaev, O. F. Vyvenko, E. V. Ubyivovk, Yu. B. Samsonenko, A. D. Bouravleuv, I. P. Soshnikov, N. V. Sibirev, G. E. Cirlin, and V. G. Dubrovskii, Phys. Status Solidi (RRL) 4, 175 (2010).

${ }^{18}$ T. B. Hoang, A. F. Moses, H. L. Zhou, D. L. Dheeraj, B. O. Fimland, and H. Weman, Appl. Phys. Lett. 94, 133105 (2009).

${ }^{19}$ S.-G. Ihn, M.-Y. Ryu, and J.-I. Song, Solid State Commun. 150, 729 (2010).

${ }^{20}$ U. Jahn, J. Lähnemann, C. Pfüller, O. Brandt, S. Breuer, B. Jenichen, M. Ramsteiner, L. Geelhaar, and H. Riechert, Phys. Rev. B 85, 045323 (2012).

${ }^{21}$ R. Gurwitz, A. Tavor, L. Karpeles, I. Shalish, W. Yi, G. Seryogin, and V. Narayanamurti, Appl. Phys. Lett. 100, 191602 (2012).

${ }^{22}$ P. Kusch, S. Breuer, M. Ramsteiner, L. Geelhaar, H. Riechert, and S. Reich, Phys. Rev. B 86, 075317 (2012).
${ }^{23}$ L. Ahtapodov, J. Todorovic, P. Olk, T. Mjåland, P. Slåttnes, D. L. Dheeraj, A. T. J. van Helvoort, B.-O. Fimland, and H. Weman, Nano Lett. 12, 6090 (2012).

${ }^{24}$ I. Zardo, S. Conesa-Boj, F. Peiro, J. R. Morante, J. Arbiol, E. Uccelli, G. Abstreiter, and A. Fontcuberta i Morral, Phys. Rev. B 80, 245324 (2009).

${ }^{25}$ Y. P. Varshni, Physica 34, 149 (1967).

${ }^{26}$ I. Vurgaftman, J. R. Meyer, and L. R. Ram-Mohan, J. Appl. Phys. 89, 5815 (2001).

${ }^{27}$ S. Cho, J. Korean Phys. Soc. 41, 475 (2002).

${ }^{28}$ P. Corfdir and P. Lefebvre, J. Appl. Phys. 112, 053512 (2012).

${ }^{29}$ Y.-H. Cho, G. H. Gainer, A. J. Fischer, J. J. Song, S. Keller, U. K. Mishra, and S. P. DenBaars, Appl. Phys. Lett. 73, 1370 (1998).

${ }^{30}$ T. Nuytten, M. Hayne, B. Bansal, H. Y. Liu, M. Hopkinson, and V. V. Moshchalkov, Phys. Rev. B 84, 045302 (2011).

${ }^{31}$ P. P. Paskov, R. Schifano, B. Monemar, T. Paskova, S. Figge, and D. Hommel, J. Appl. Phys. 98, 093519 (2005).

${ }^{32}$ P. Corfdir, P. Lefebvre, J. Levrat, A. Dussaigne, J.-D. Ganière, D. Martin, J. Ristić, T. Zhu, N. Grandjean, and B. Deveaud-Plédran, J. Appl. Phys. 105, 043102 (2009).

${ }^{33}$ S. Yang, C. C. Kuo, W.-R. Liu, B. H. Lin, H.-C. Hsu, C.-H. Hsu, and W. F. Hsieh, Appl. Phys. Lett. 100, 101907 (2012).

${ }^{34}$ S. Hammersley, D. Watson-Parris, P. Dawson, M. J. Godfrey, T. J. Badcock, M. J. Kappers, C. McAleese, R. A. Oliver, and C. J. Humphreys, J. Appl. Phys. 111, 083512 (2012).

${ }^{35}$ C. Weisbuch, R. Dingle, A. C. Gossard, and W. Wiegmann, Solid State Commun. 38, 709 (1981).

${ }^{36}$ R. Zimmermann, F. Grosse, and E. Runge, Pure Appl. Chem. 69, 1179 (1997).

${ }^{37}$ V. Srinivas, Y. J. Chen, and C. E. C. Wood, Solid State Commun. 89, 611 (1994).

${ }^{38}$ F.-Y. Juang, Y. Nashimoto, and P. K. Bhattacharya, J. Appl. Phys. 58, 1986 (1985).

${ }^{39}$ P. B. Kirby, J. A. Constable, and R. S. Smith, J. Appl. Phys. 69, 517 (1991).

${ }^{40}$ P. Corfdir, P. Lefebvre, J. Ristić, J. D. Ganière, and B. DeveaudPlédran, Phys. Rev. B 80, 153309 (2009).

${ }^{41}$ D. Gammon, S. Rudin, T. L. Reinecke, D. S. Katzer, and C. S. Kyono, Phys. Rev. B 51, 16785 (1995).

${ }^{42}$ S. Rudin, T. L. Reinecke, and B. Segall, Phys. Rev. B 42, 11218 (1990).

${ }^{43}$ M. Brewster, O. Schimek, S. Reich, S. Gradečak, Phys. Rev. B 80, 201314(R) (2009).

${ }^{44}$ D. Strauch and B. Dorner, J. Phys. Condens. Matter 2, 1457 (1990).

${ }^{45}$ L. Schultheis, A. Honold, J. Kuhl, K. Kohler, and C. W. Tu, Phys. Rev. B 34, 9027 (1986).

${ }^{46}$ D.-S. Kim, J. Shah, J. E. Cunningham, T. C. Damen, W. Schäfer, M. Hartmann, and S. Schmitt-Rink, Phys. Rev. Lett. 68, 1006 (1992).

${ }^{47}$ W. Braun, M. Bayer, A. Forchel, H. Zull, J. P. Reithmaier, A. I. Filin, and T. L. Reinecke, Phys. Rev. B 56, 12096 (1997).

${ }^{48}$ C.-Y. Yeh, S.-H. Wei, and A. Zunger, Phys. Rev. B 50, 2715 (1994).

${ }^{49}$ C. Piermarocchi, F. Tassone, V. Savona, A. Quattropani, and P. Schwendimann, Phys. Rev. B 53, 15834 (1996).

${ }^{50}$ H. Hillmer, A. Forchel, S. Hansmann, M. Morohashi, E. Lopez, H. P. Meier, and K. Ploog, Phys. Rev. B 39, 10901 (1989). 
${ }^{51}$ P. Borri, W. Langbein, J. M. Hvam, and F. Martelli, Phys. Rev. B 59, 2215 (1999).

${ }^{52}$ F. Sajovec, R. Wolf, A. Fattah, K. Bickmann, H. Wenzl, G. Nagel, H. Rüfer, E. Tomzig, and P. de Bièvre, Phys. Status Solidi A 122, 139 (1990).

${ }^{53}$ J. He, B. Djafari-Rouhani, and J. Sapriel, Phys. Rev. B 37, 4086 (1988).
${ }^{54}$ P. Pfeffer, I. Gorzyca, and W. Zawadzki, Solid State Commun. 51, 179 (1984).

${ }^{55}$ T. Cheiwchanchamnangij and W. R. L. Lambrecht, Phys. Rev. B 84, 035203 (2011).

${ }^{56}$ A. De and C. E. Pryor, Phys. Rev. B 81, 155210 (2010).

${ }^{57}$ W. Peng, B. Jusserand, J. C. Harmand, and M. Bernard, Appl. Phys. Lett. 100, 073102 (2012). 